\title{
A Multi-Objective Coordinated Charging and Discharging Strategy for Electric Vehicles Based on Stackelberg Game
}

\author{
Haozhe Xu, Xueliang Huang \\ School of Electrical Engineering, Southeast University, Nanjing, China \\ Email:220172713@seu.edu.cn
}

How to cite this paper: $\mathrm{Xu}, \mathrm{H} . \mathrm{Z}$. and Huang, X.L. (2020) A Multi-Objective Coordinated Charging and Discharging Strategy for Electric Vehicles Based on Stackelberg Game. Energy and Power Engineering, 12, 63-72.

https://doi.org/10.4236/epe.2020.124B007

Received: March 11, 2020

Accepted: April 7, 2020

Published: April 10, 2020

\begin{abstract}
For the negative impact of large-scale electric vehicles (EVs) disorderly charging on the power grid, a multi-objective optimization strategy for coordinated charging and discharging of EVs based on Stackelberg game is proposed. As the leader, the grid company aims to stabilize load fluctuations and formulate a reasonable electricity price strategy to guide EVs to participate in vehicle-to-grid (V2G); As followers, EV users optimize their charging plans based on electricity price information with the objective of reducing costs and obtaining good comfort. This paper uses the MOPSO algorithm to solve the proposed multi-objective Stackelberg problem, and calculates the optimization results under various preferences, which proves the effectiveness of the pro-posed model and method.
\end{abstract}

\section{Keywords}

Vehicle-to-Grid (V2G), Stackelberg Game, Electric Vehicle (EV), Optimal Dispatch

\section{Introduction}

With the increasingly serious problems of global environmental pollution and energy shortage, electric vehicle with the characteristics of environmental protection and energy saving has been widely concerned and rapidly developed. The increase in the number of EVs and their uncoordinated charging will cause problems such as grid overload, increased network losses, and harmonic pollution [1] [2] [3], which is not conducive to the security and stability of the power grid. V2G uses the mobile energy storage characteristic of EVs to rationally regulate the charging and discharging of EVs, which can not only alleviate fluctua- 
tions in the load on the power grid, but also create revenue for EV users [4].

The Stackelberg problem with the hierarchical structure was first raised by Von Stackelberg in 1952 when he studied market economy issues [5]. In [6], a Stackelberg game approach was used to deal with the charging strategy in the case of one aggregator and multiple aggregators. Similar approaches were used in [7] to stimulate the game between community charging agents and EVs, by transforming the game into linear programming, the pricing and power purchasing strategies of smart community agents were obtained. In [8], a Stackelberg game model is established to study the V2G problem in the parking lot and the battery loss and transmission loss are considered to optimize the robust characteristics of the system. Additionally, a charging guidance method based on electricity price signals.is proposed in [9] to maximize EVs benefits and to eventually flatten aggregated load curves. The work in [10] considered the uncertainty of EV behavior during the game, and proposed a real-time asynchronous DR algorithm, which effectively reduced the load fluctuation of the power grid. A three-layer Stackelberg model including power grid, energy storage, and users is constructed in [11], and the Jaya-based Boltzmann machine algorithm was used to maximize the overall benefits of the game players.

The Stackelberg model in the above literature only considers the single objective of each subject, and in reality, the objectives of the game players are often diversified. Especially for EVs, only considering economic objective may ignores the user's requirement for comfort. Based on the previous research, this paper builds a Stackelberg multi-objective optimization model with the grid as the leader and the EV users as the followers. The MOPSO algorithm is used to calculate the charging and discharging strategies under different preferences. Main contributions of this paper include following key points:

- Using a Stackelberg game between grid and EVs and considering their multiple objectives to make the model closer to reality.

- Designing examples with different preferences, and finding Stackelberg equilibrium with MOPSO algorithm and fuzzy theory.

The remaining of this paper is organized as follows: Section 2 discusses the system model and formulates the Stackelberg problem. In Section 3, the solution techniques adopted in this work have been discussed. The results obtained from our simulations are provided and discussed in Section 4 . Section 5 concludes the paper

\section{System Model}

As shown in Figure 1, the power grid is upper-level leader, and EVs are lower-level followers. EV users adjust their charge and discharge plans to respond to electricity price signals from the power grid in order to reduce the cost of electricity, but they will also consider their original charging plan. Different types of EVs have different behavior characteristics and power requirements [12]. The research object of this article is private $\mathrm{EV}$. 


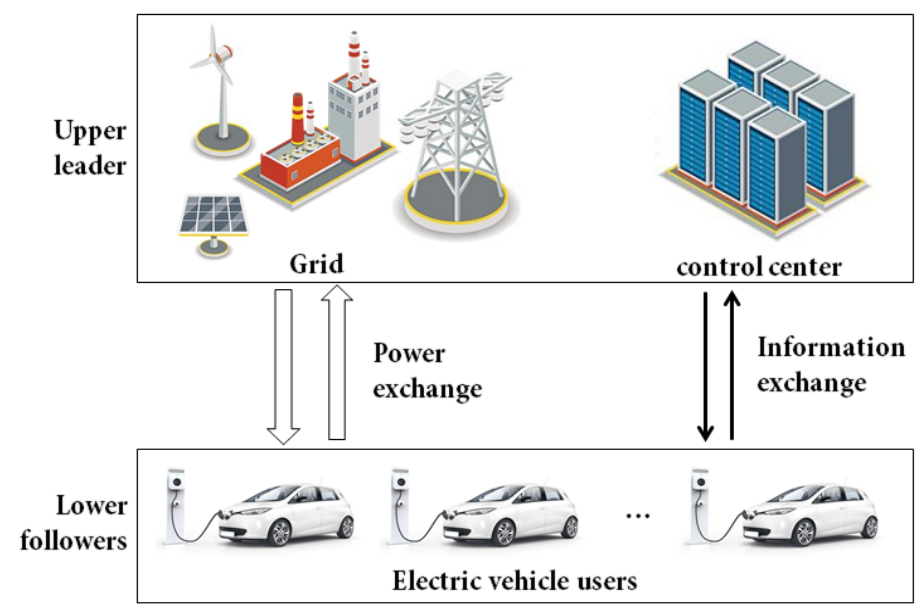

Figure 1. V2G system model.

\subsection{User Model}

According to what has been s explained in Section 1, EV users participate in V2G and formulate their own charging and discharging plans to reduce charging costs. On the other hand, they also want to obtain good experience of consuming. Therefore, the objectives of EV users should include the two aspects of economy and comfort. The user problem can be formulated as follows:

- Objective 1: Optimal economy

$$
\min U_{E V 1, i}=\sum_{t=1}^{T}\left(p_{t} \cdot x_{i, t} \cdot \Delta t+K_{d} \cdot\left|x_{i, t}\right| \cdot \Delta t\right) .
$$

where $x_{i, t}$ is the charging or discharging power of EV i during the time interval $\mathrm{t}, x_{i, t}>0$ means charging and $x_{i, t}<0$ means discharging. $p_{t}$ is the electricity price in the interval t. $K_{d}$ is the battery discount rate. Factors affecting the discount rate include battery discharge depth, charge and discharge power, temperature, etc. [13] [14].

- Objective 2: Optimal Comfort

The comfort of EV users is closely related to their charging plans. When there is no difference in electricity prices in different time periods and the user has no economic considerations, the user will choose the charging plan that best meets his own habits. Therefore, the user's load curve without the difference in electricity prices can be used as the maximum comfort curve, and the deviation between the actual load curve and the maximum comfort curve can be used as an index to measure the electric comfort of the EV user. Therefore, the optimization problem is formulated as follows:

$$
\max U_{E V 2, i}=1-\frac{\sum_{t=1}^{T}\left|\alpha_{t}\left(x_{i, t}-x_{i, t, 0}\right)\right|}{\sum_{t=1}^{T} x_{i, t, 0}} .
$$

where, $x_{i, t, 0}$ is the original planned load of EV $\mathrm{i}$ in the interval $\mathrm{t}$, and it depends on the driving habits and travel characteristics of the user. It can be simulated by 
Monte Carlo method after fitting the relevant statistical data [15] [16] [17]. $\alpha_{t}$ is the influence coefficient, which measures the influence of the deviation on the user's comfort. A small value can be set at night and a large value can be set during the day.

- The constraints of users

$$
\begin{gathered}
\text { s.t. } \quad x_{\text {min }} \leq x_{i, t} \leq x_{\text {max }} . \\
S O C_{\text {min }} \leq S O C_{i, t} \leq S O C_{\text {max }} . \\
S O C_{i, t}=S O C_{i, t-1}+\frac{x_{i, t}}{C_{0}} \cdot \Delta t . \\
S O C_{i, t \text { (departure) }}=S O C_{\text {max }} .
\end{gathered}
$$

where (3) provides the upper and lower boundaries for charge and discharge power of EV and (4) gives the upper and lower boundaries of the battery SOC (State of Charge). (5) shows the relationship between the current SOC and the SOC of the previous period. In (6) the sequence of charging/discharging should be in a way that by the time that the EV owner decides to leaves the house, the SOC should reached maximum.

\subsection{Grid Model}

The objectives of the power grid include reducing the standard deviation of the load, reducing the peak-to-valley difference, and increasing the revenue from electricity sales. Some literatures [18] [19] [20] have studied the economic benefits of V2G to the grid, i.e., the effect of subsidizing EVs to participate in V2G is conducive to the reduction of the overall cost of the grid. Therefore, we no longer consider the objective of maximizing the electricity sales revenue of the power grid, and only considers the index of load stability.

- Objective 1: Reduce standard deviation of the load

$$
\begin{aligned}
\min U_{\text {grid1 }} & =\sqrt{\frac{1}{T} \sum_{t=1}^{T}\left(L_{t}-\bar{L}\right)^{2}} . \\
L_{t} & =L_{0, t}+\sum_{i=1}^{I} x_{i, t} . \\
\bar{L} & =\frac{1}{T} \cdot \sum_{t=1}^{T} L_{t} .
\end{aligned}
$$

where $L_{0, t}$ is the power of grid without EV load in the interval t. $L_{t}$ is the total load in the interval t. $\bar{L}$ is the daily average load after scheduling.

- Objective 2: Reduce peak-to-valley differences

$$
\min U_{\text {grid } 2}=L_{\max }-L_{\min } \text {. }
$$

where, $L_{\max }$ is the daily maximum load, $L_{\min }$ is the daily minimum load.

- The constraints of grid

$$
\begin{aligned}
\text { s.t. } \quad p_{\min }<p_{t}<p_{\max } . \\
L_{t} \leq M .
\end{aligned}
$$


where (16) provides the upper and lower boundaries for electricity price in each time period. (17) limits the maximum value of the total load and it cannot exceed the maximum capacity of the distribution network.

\section{Stackelberg Game Model Solution}

The original load curve of an EV depends on the owner's behavior, the battery parameters of the EV, etc. The relevant statistics of the 2009 National Household Travel Survey (NHTS) [21] are shown in Figure 2. The daily charging time of the EV is obtained through the calculation of the daily driving distance, the power consumption of 100 kilometers, and the charging power. In combination with the moment when EVs start charging, Monte Carlo simulation can be used to obtain the original charging load when the EV did not participate in V2G.

This paper uses the multi-objective particle swarm optimization (MOPSO) algorithm [22] to solve the multi-objective Stackelberg model. The MOPSO algorithm searches for the optimal solution by continuously updating its position and speed to obtain a set of Pareto optimal solution. After obtaining the Pareto optimal set, the method of fuzzy theory [23] is used to select the optimal solution. The objective function value of a non-inferior solution in the solution set is fuzzified by membership function.

$$
\mu_{k}^{N}=\frac{f_{k}^{\max }-f_{k}^{n}}{f_{k}^{\max }-f_{k}^{\min }} .
$$

where $f_{k}^{n}$ is the $\mathrm{K}$-th objective function value of non-inferior solution $x_{n}$. $f_{k}^{\max }$ and $f_{k}^{\min }$ are the maximum and minimum values of the $\mathrm{k}$-th objective function. After weighted summing according to the objective preference, the comprehensive satisfaction $\mu^{n}$ can be obtained.

$$
\mu^{n}=\frac{\sum_{k=1}^{K} \theta_{k} \mu_{k}^{n}}{\sum_{n=1}^{N} \sum_{k=1}^{K} \theta_{k} \mu_{k}^{n}} .
$$

where $K$ is the total number of objectives, $N$ is the total number of non-inferior solutions, and $\theta_{k}$ is the weight of the k-th objective.

In Figure 3, MOPSO algorithm is illustrated via a flow chart.
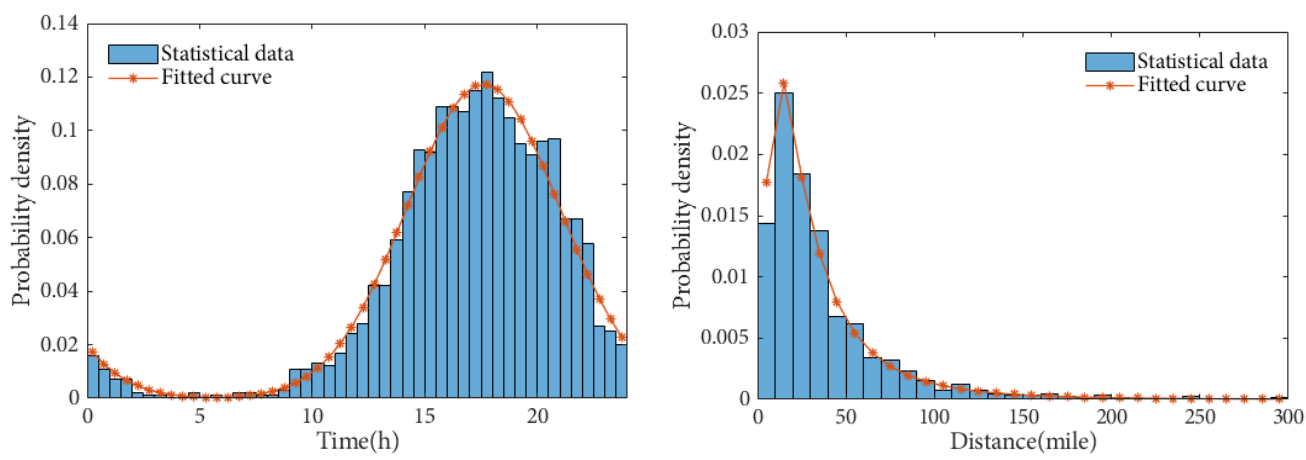

Figure 2. The moment when EVs start charging \& daily travel distance of EVs. 


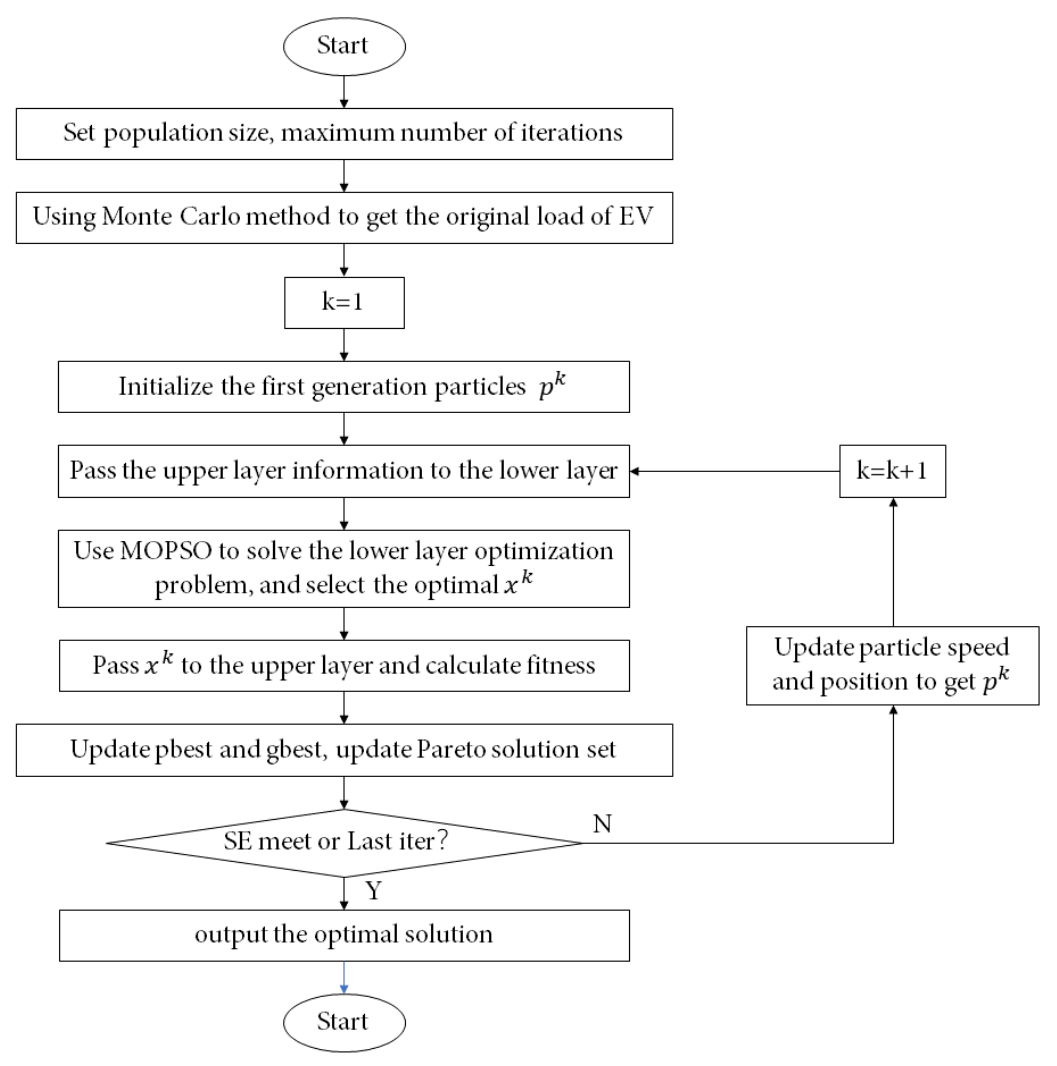

Figure 3. Stackelberg game algorithm.

\section{Simulation Result}

This paper uses 1000 EVs in an area as an example for calculation. Relevant research shows that [24], the average daily driving time of more than $90 \%$ of private cars is only about one hour and is generally not in the peak and valley hours of the power grid. The cars are idle in $95 \%$ of the time. On the other hand, in the future, centralized charging piles and V2G equipment can be built in the parking lot for centralized charging, which can ensure a higher connection rate for EVs. Therefore, it can be considered that EVs can participate in V2G optimized scheduling within 24 hours. The relevant parameter settings are shown in Table 1.

The typical daily load curve of an area and the disordered charging load of an EV obtained by Monte Carlo simulation are shown in Figure 4. It can be seen that the uncoordinated charging of EVs will increase the peak-to-valley difference of the total load, and the load fluctuation will be more serious, which will adversely affect the security and stability of the grid.

\subsection{Strategy A: Considering Only Economy}

Without considering the comfort of power consumption, i.e., the lower layer $\theta_{1}=1, \theta_{2}=0$, and set the upper layer $\theta_{1}=0.5, \theta_{2}=0.5$. According to the above algorithm, the optimization results are shown in Figure 5.

From the simulation results, it can be known that under the strategy considering only Economy, the load of EVs is sensitive to changes in electricity prices, 
Table 1. Simulation parameters.

\begin{tabular}{cccc}
\hline$x_{\min }(k W)$ & 0 & $S O C_{\min }$ & 0.2 \\
$x_{\max }(k W)$ & 7 & $S O C_{\max }$ & 0.9 \\
$C_{0}(k W h)$ & 57 & $T($ departure $)$ & 7 \\
$K_{d}($ yuan $/ k W h)$ & 0.12 & $p_{\text {min }}($ yuan $/ k W h)$ & 0.3 \\
$M(M W)$ & 100 & $p_{\text {max }}($ yuan $/ k W h)$ & 0.8 \\
\hline
\end{tabular}

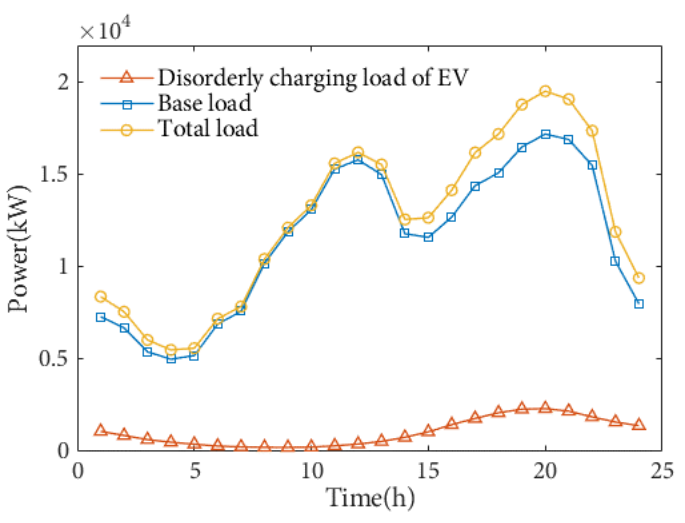

Figure 4. Uncoordinated charging load.
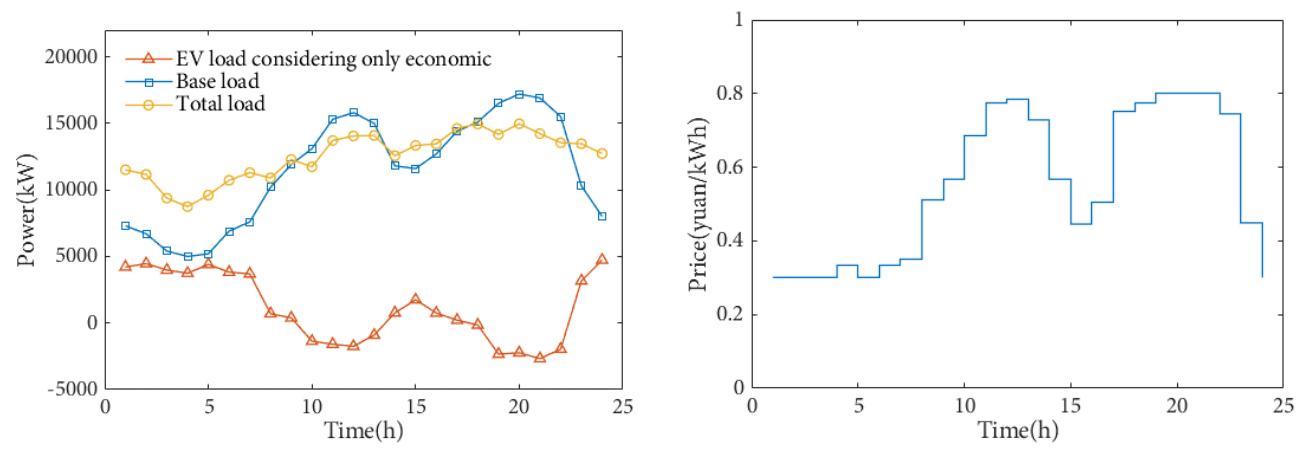

Figure 5. Load and electricity price in strategy A.

and they can effectively adjust the charge and discharge load. The effect of peak-cutting and valley-filling is obvious. After optimization, the standard deviation of the total load of the power grid has been reduced by $60.06 \%$ compared to that of uncoordinated charging, the peak-to-valley difference has been reduced by $55.61 \%$ and the total charging cost has been reduced by $14,889.1$ yuan. However, in this case, the comfort level of electricity consumption is low, only -0.26 . The electricity price shows obvious peak-to-valley characteristics, making full use of the owner's pursuit of economic benefits, and effectively guiding the users to orderly charge and discharge.

\subsection{Strategy B: Considering Only Comfort}

According to the analysis in section 2.1, if the user only considers the comfort and does not consider the economy, the user's comfort is the best when the user 
does not change his own charging plan. In this case, the load of the EV is consistent with the original uncoordinated charging. The comfort level is the highest, but the economy is the worst.

\subsection{Strategy C: Considering Economy and Comfort}

The lower layer $\theta_{1}=0.5, \theta_{2}=0.5$, and the upper layer $\theta_{1}=0.5, \theta_{2}=0.5$. The optimization results are shown in Figure 6.

After optimization, the standard deviation of the load is reduced by $49.44 \%$, the peak-to-valley difference is reduced by $46.42 \%$, and the total charging cost is reduced by $11,285.1$ yuan. At this time, the comfort is -0.06 , which is better than considering only economy. Compared with the strategy only considering economy, the strategy that comprehensively considers economy and comfort has a smaller electricity price difference. Because users have both economic and comfort objectives, more adjustments to charging and discharging may reduce overall satisfaction, resulting in low user response to electricity prices. Therefore, the higher electricity price difference has no obvious effect.

The data of the three strategies are shown in Table 2.

\section{Conclusions}

This paper builds a multi-objective Stackelberg game model to study the optimization strategy of EVs and power grids in terms of energy exchange and electricity pricing. The following conclusions are obtained:

1) Electric vehicles can effectively reduce their costs by participating in V2G, and the grid can guide users to orderly charge and discharge by setting appropriate electricity price to achieve the purpose of peak cutting and valley filling.
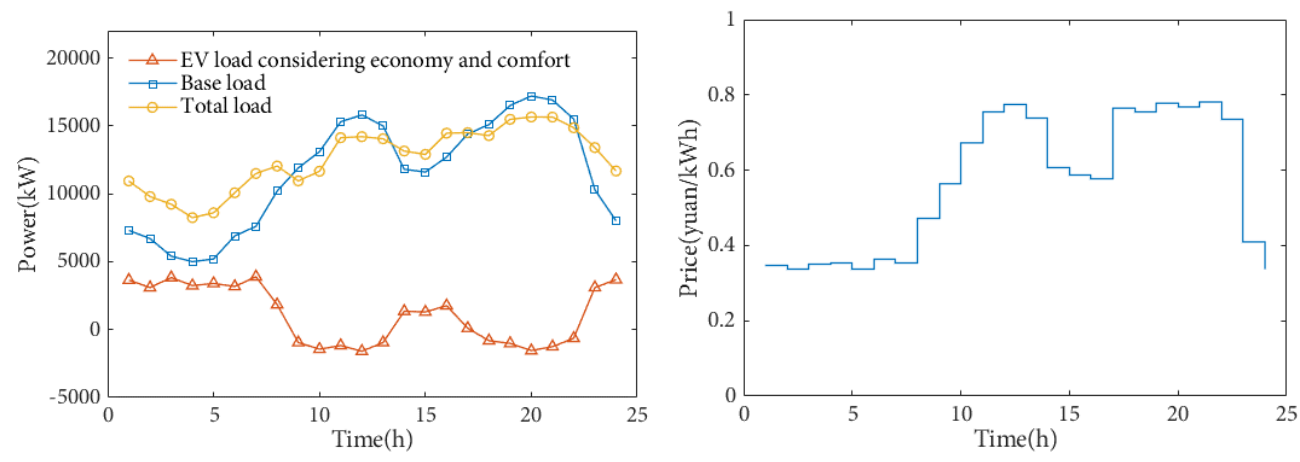

Figure 6. Load and electricity price in strategy C.

Table 2. Data of three strategies.

\begin{tabular}{cccccc}
\hline Data & $\begin{array}{c}\text { Standard } \\
\text { deviation of load }\end{array}$ & $\begin{array}{c}\text { Peak-valley } \\
\text { difference of load }\end{array}$ & $\begin{array}{c}\text { Users } \\
\text { cost }\end{array}$ & $\begin{array}{c}\text { Users } \\
\text { comfort }\end{array}$ & $\begin{array}{c}\text { Price(max)/pri } \\
\text { ce(min) }\end{array}$ \\
\hline Strategy A & 1808.7 & 6224.3 & 2513.7 & -260 & 2.67 \\
Strategy B & 4528.1 & $14,023.3$ & $17,402.8$ & 1000 & 1 \\
Strategy C & 2289.6 & 7513.0 & 6117.7 & -60 & 2.31 \\
\hline
\end{tabular}


2) Under the strategy considering only Economy, the user's economic benefits are the best but the power comfort is the worst.

3) Under the strategy considering only Comfort, users charge disorderly, the comfort level is the best but the economy is the worst, and the load fluctuation of the power grid increases.

4) The strategy considering Economy and Comfort can reduce the load fluctuation of the grid and reduce the power cost of EV users. At the same time, it ensures a certain level of comfort, and the data in various aspects are relatively balanced.

\section{Conflicts of Interest}

The authors declare no conflicts of interest regarding the publication of this paper.

\section{References}

[1] Ma, L.-L., Yang, J., Fu, C., et al. (2013) Review on Impact of Electric Car Charging and Discharging on Power Grid. Power System Protection and Control, No. 3, 140-148.

[2] Gao, C.W. and Zhang, L. (2011) A Survey of Influence of Electrics Vehicle Charging on Power Grid. Power System Technology, No. 2, 133-137.

[3] He, J.H., Xie, Y.Y., Yip, T., Wang, X.J. and Li, Z.C. (2015) Influence of Electric Vehicles Charging Modes on Active Network Distribution. Electric Power Construction, 36, 97-102.

[4] Liu, X.F., Zhang, Q.F. and Cui, S.M. (2012) Review of Electric Vehicle V2G Technology, Transactions of China Electrotechnical Society, 27, 121-127.

[5] Kaldor, N. (1936) Reviewed Work: Marktform und Gleichgewicht by H. von Stackelberg. Economica, 3, 227. https://doi.org/10.2307/2549070

[6] Shinde, P. and Shanti Swarup, K. (2018) Stackelberg Game-Based Demand Response in Multiple Utility Environments for Electric Vehicle Charging. IET Electrical Systems in Transportation, 8, 167-174, 9. https://doi.org/10.1049/iet-est.2017.0046

[7] Wei, W., Chen, Y., Liu, F., Mei, S.W., Tian, F. and Zhang, X. (2015) Stackelberg Game Based Retailer Pricing Scheme and EV Charging Management in Smart Residential Area. Power System Technology, 39, 939-945.

[8] Guo, D. and Zhou, C. (2018) Realistic Modeling of Vehicle-to-Grid in an Enterprise parking Lot: A Stackelberg Game Approach. 2018 IEEE Texas Power and Energy Conference (TPEC), College Station, TX, 1-6. https://doi.org/10.1109/TPEC.2018.8312099

[9] Shi, Y., Feng, D., Zhou, E. and Chen, F. (2019) Stackelberg Game Based on Supervised Charging Method and Pricing Strategy of Charging Service Providers. Transactions of China Electrotechnical Society, 34, 742-751.

[10] Azimian, B., Fijani, R.F., Ghotbi, E. and Wang, X. (2018) Stackelberg Game Approach on Modeling of Supply Demand Behavior Considering BEV Uncertainty. 2018 IEEE International Conference on Probabilistic Methods Applied to Power Systems (PMAPS), Boise, ID, 1-6. https://doi.org/10.1109/PMAPS.2018.8440398

[11] Samuel, O., Khan, Z.A., Iqbal, S. and Javaid, N. (2018) An Efficient Energy Management in Microgrid: A Game Theoretic Approach. 2018 Fifth HCT Information 
Technology Trends (ITT), Dubai, United Arab Emirates, 205-212. https://doi.org/10.1109/CTIT.2018.8649492

[12] Chen, J. (2018) Study of the Charging Load Characteristics and Orderly Charging Strategy of EVs. Wuhan University, Wuhan.

[13] Liu, L., Liu, T., Zhang, T. and Liu, J. (2016) Orderly Charging and Discharging Strategy Optimization for Electric Vehicles Considering Dynamic Battery-Wear Model. Automation of Electric Power Systems, 40, 83-90.

[14] Lu, L., Wen, F., Xue, Y. and Kang, J. (2013) Economic Analysis of Ancillary Service Provision by Plug-in Electric Vehicles. Automation of Electric Power Systems, 37, 43-49+58.

[15] Qian, K., Zhou, C., Allan, M. and Yuan, Y. (2011) Modeling of Load Demand Due to EV Battery Charging in Distribution Systems. IEEE Transactions on Power Systems, 26, 802-810. https://doi.org/10.1109/TPWRS.2010.2057456

[16] Tian, L., Shi, S. and JIa, Z. (2010) A Statistical Model for Charging Power Demand of Electric Vehicles. Power System Technology, 34, 126-130.

[17] Li, H., Du, Z., Chen, L., Guan, L. and Zhou, B. (2019) Trip Simulation Based Charging Load Forecasting Model and Vehicle-to-Grid Evaluation of Electric Vehicles. Automation of Electric Power Systems, 43, 88-102.

[18] Nworgu, O.A., Chukwu, U.C., Okezie, C.G. and Chukwu, N.B. (2016) Economic Prospects and Market Operations of V2G in Electric Distribution Network. 2016 IEEE/PES Transmission and Distribution Conference and Exposition (T\&D), Dallas, TX, 1-5. https://doi.org/10.1109/TDC.2016.7519938

[19] Almehizia, A.A. and Snodgrass, J.M. (2018) Investigation of V2G Economical Viability. 2018 IEEE Texas Power and Energy Conference (TPEC), College Station, TX, 1-6. https://doi.org/10.1109/TPEC.2018.8312048

[20] Shafiq, S., Akram, U., Awami, A.T.A. and Al-Muhaini, M. (2016) Reliability Evaluation and Economic Assessment of Micro-Grid with V2G Electric Vehicles Coordination. 2016 Saudi Arabia Smart Grid (SASG), Jeddah, 1-7. https://doi.org/10.1109/SASG.2016.7849664

[21] U.S. Department of Transportation (2009) 2009 National Household Travel Survey.

[22] Coello, C.A.C., Pulido, G.T. and Lechuga. M.S. (2004) Handling Multiple Objectives with Particle Swarm Optimization. IEEE Transactions on Evolutionary Computation, 8, 256-279. https://doi.org/10.1109/TEVC.2004.826067

[23] Agrawal, S., Panigrahi, B.K. and Tiwari, M.K. (2008) Multiobjective Particle Swarm Algorithm with Fuzzy Clustering for Electrical Power Dispatch. IEEE Transactions on Evolutionary Computation, 12, 529-541. https://doi.org/10.1109/TEVC.2007.913121

[24] Wei, D., Zhang, C., Sun, B. and Cui, N. (2014) A Time-of-Use Price Based Multi-Objective Optimal Dispatching for Charging and Discharging of Electric Vehicles. Power System Technology, 38, 2972-2977. 